For Internal Distribution Only

Accelerator Division

Alternating Gradient Synchrotron Department BROOKHAVEN NATIONAL LABORATORY

Upton, New York 11973

Accelerator Division

Technical Note

AGS/AD/Tech. Note No. 446

\title{
A VERTICAL BUMP AT THE J-10 SCRAPER
}

\author{
E.J. Bleser
}

October 9, 1996 


\title{
A VERTICAL BUMP AT THE J-10 SCRAPER
}

\author{
E.J. Bleser
}

\section{SUMMARY}

This note describes a possible vertical bump at the J-10 scraper using the new steering dipoles. At injection this bump can be strong enough to scrape off most of the beam.

\section{INTRODUCTION}

The beam scraper to be installed at J-10 in the summer of 1997 will be essentially fixed in position, and the beam will be moved onto it using local orbit bumps. At present, we have significant injection losses at vertical apertures in the ring, and our goal is to collect those losses on the scraper. This note finds that a local vertical bump using the new low field steering magnet system will scrape the injected beam very nicely. Similar bumps might be designed to scrape the beam at the E-20 dump for the winter 1997 run.

\section{THE 3-BUMPS}

This note documents two basic three-bump designs: the short three-bump and the long threebump. The short three-bump uses three dipoles in sequence. The long three-bump uses magnets 1 , 3 , and 4 in a sequence of four magnets. Table 1 gives the phase advances between various dipoles for "The Standard Injection AGS" (Appendix I). By skipping the second magnet in the sequence, there is always more than a 90 degree phase advance between the first and second magnets of the bump. Therefore at the second magnet of the bump the orbit has already peaked and is decreasing which means that the second magnet of the bump must just steer the orbit back to the axis at the third magnet of the bump. Thus for long three-bumps the first magnet is the strongest magnet and determines the peak amplitude. For short three-bumps this is not always true and the achievable amplitude is less than for the long three-bump. In a superperiod the new steering dipoles will be located in straight sections $2,8,12$, and 18. Thus for each kind of bump there are four variations. The bumps will be named by the initial magnet position along with the suffix $S$ for short bump or $\mathrm{L}$ for long bump. The required dipole strengths are readily calculated from the formula:

$$
\frac{\theta_{1} \sqrt{\beta_{1}}}{\sin \left(\Delta \mu_{3,2}\right)}=\frac{\theta_{2} \sqrt{\beta_{2}}}{\sin \left(\Delta \mu_{1,3}\right)}=\frac{\theta_{3} \sqrt{\beta_{3}}}{\sin \left(\Delta \mu_{2,1}\right)}
$$

where $\theta$ is the bend and $\Delta \mu_{\mathrm{m}, \mathrm{n}}$ is the phase at $\mathrm{m}$ minus the phase at $\mathrm{n}$. Table 2 gives the bends calculated from this formula. At injection the maximum dc current is 12.5 Amperes which gives a bend of $1 / 3$ of a milliradian. Scaling the bends in Table 2 by this value and feeding them into 
MAD gives orbits plotted in Figures 1 through 4 and summarized in Table 2 . It should be noted that bumps calculated from the formula above have almost no residuals in the MAD calculations.

\section{COMBINING BUMPS}

In order to maximize the bump amplitude at the J-10 Scraper we combine two long bumps, J02L and J08L. The results are shown in Figure 5. The second peak at J-15 is undesirable and can be reduced by subtracting bump J12L. There is a trade off here. Too much reduction at J-15 produces an undershoot at J-18 which may or may not matter. For discussion purposes we take the vertical bump for scraping at J-10 to be the combination of J02L, J08L, and J12L summarized in Table 3 and plotted in Figure 5. This bump is probably the most compact bump of significant amplitude that we can readily achieve. Table 4 records all the various bump orbits at the exits of the AGS main magnets for a peak bend of 1/3 milliradians.

Acknowledgments

This work was made possible solely through the efforts of those who have developed and maintained the MAD Program.

TABLE 1

PHASE ADVANCES BETWEEN VERTICAL DIPOLES IN STANDARD INJECTION AGS

\begin{tabular}{|c|r|r|}
\hline DIPOLE & $\begin{array}{c}\text { PHASE ADVANCE } \\
\text { FROM DIPOLE DVCJ02 } \\
\text { DEGREES }\end{array}$ & $\begin{array}{c}\text { PHASE ADVANCE } \\
\text { BETWEEN DIPOLES } \\
\text { DEGREES }\end{array}$ \\
\hline DVCJ02 & 0 & \\
\hline DVCJ08 & 84.24 & 84.24 \\
\hline DVCJ12 & 133.56 & 49.32 \\
\hline DVCJ18 & 213.12 & 79.56 \\
\hline DVCK02 & 266.76 & 53.64 \\
\hline DVCK08 & 351.00 & 84.24 \\
\hline DVCK12 & 400.32 & 49.32 \\
\hline DVCK18 & 479.88 & 79.56 \\
\hline
\end{tabular}


TABLE 2

\section{GENERIC VERTICAL THREE-BUMPS}

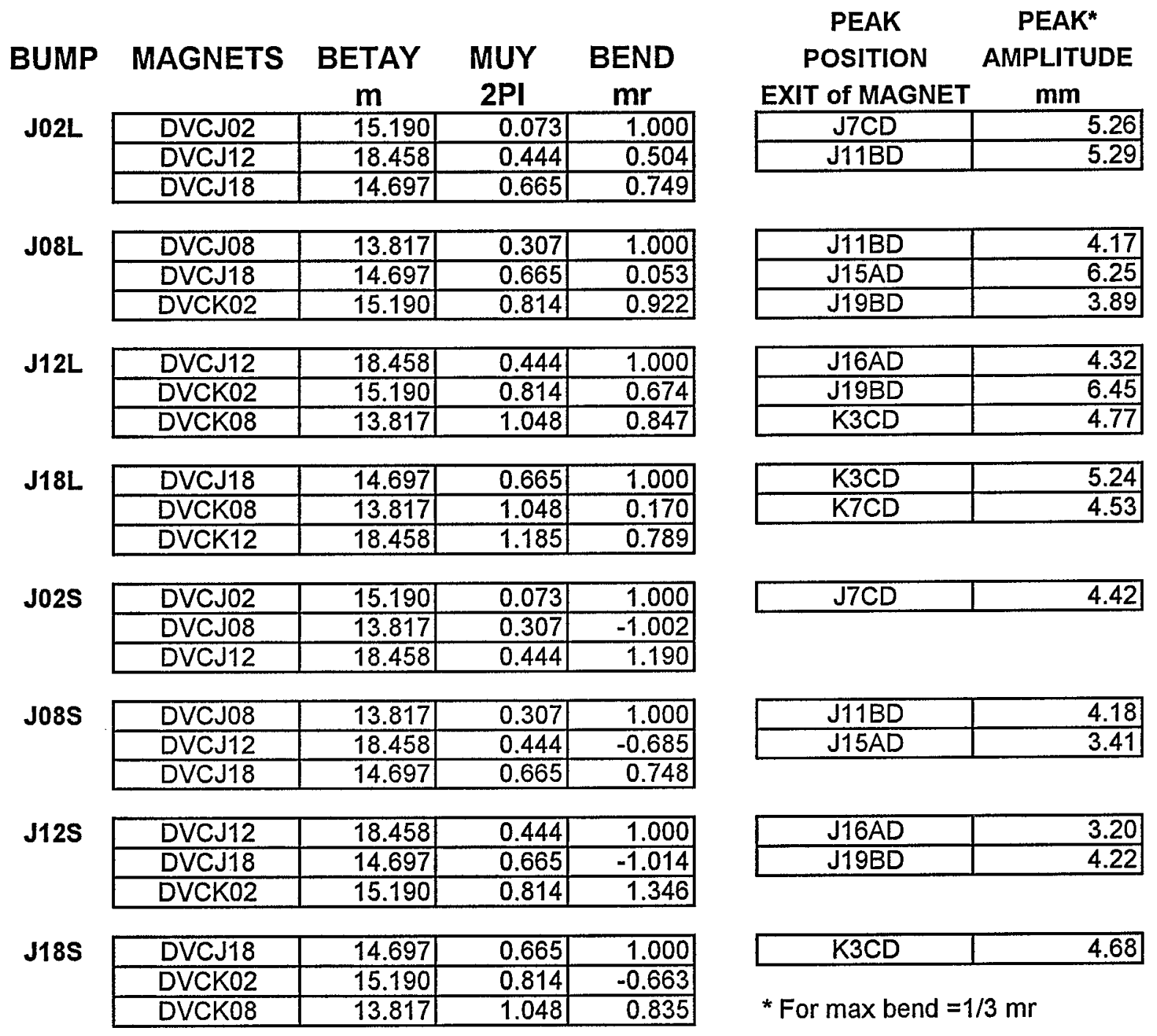


TABLE 3

THE J-10 VERTICAL BUMP

\begin{tabular}{|c|c|c|c|c|c|}
\cline { 5 - 6 } & \multicolumn{2}{l}{} & J02L+ & J02L+J08L \\
\hline MAGNETS & J02L & J08L & J12L & J08L & - J12L \\
\hline & $\mathrm{mr}$ & $\mathrm{mr}$ & $\mathrm{mr}$ & $\mathrm{mr}$ & $\mathrm{mr}$ \\
\hline DVCJ02 & 1.000 & & & 1.000 & 1.000 \\
\hline DVCJ08 & & 1.000 & & 1.000 & 1.000 \\
\hline DVCJ12 & 0.504 & & 1.000 & 0.504 & -0.496 \\
\hline DVCJ18 & 0.749 & 0.053 & & 0.802 & 0.802 \\
\hline DVCK02 & & 0.922 & 0.674 & 0.922 & 0.248 \\
\hline DVCK08 & & & 0.847 & & -0.847 \\
\hline
\end{tabular}

PEAK ORBIT AMPLITUDES

for $1 / 3 \mathrm{mr}$ maximum bend

AT THE EXIT OF MAGNET
\begin{tabular}{|c|r|r|r|r|r|}
\hline BUMP & \multicolumn{1}{|c|}{ J7CD } & J11CD & J15BE & \multicolumn{1}{|c|}{ K3CD } & \\
\hline J02L+J08L & 5.27 & 9.45 & 9.670 & 0.000 & $\mathrm{~mm}$ \\
\hline J02L+J08L-J12L & 5.25 & 9.46 & 5.500 & -4.790 & $\mathrm{~mm}$ \\
\hline
\end{tabular}


TABLE 4

\section{SUMMARY of VERTICAL BUMP ORBITS}

\begin{tabular}{|c|c|c|c|c|c|c|c|c|c|}
\hline & & J02L & J02S & J08L & J08S & J12L & J12S & J18L & J18S \\
\hline EXIT of & $\bar{s}$ & $\overline{\mathrm{Y}}$ & $\bar{Y}$ & $\bar{Y}$ & $\bar{Y}$ & $\bar{Y}$ & $\bar{Y}$ & $\bar{Y}$ & $\bar{Y}$ \\
\hline MAGNET & meters & $\mathrm{mm}$ & $\mathrm{mm}$ & $\mathrm{mm}$ & $\mathrm{mm}$ & $\mathrm{mm}$ & $\mathrm{mm}$ & $\mathrm{mm}$ & $\mathrm{mm}$ \\
\hline J1BF & 607.31 & -0.01 & -0.01 & & & & & & \\
\hline J2BF & 609.93 & -0.01 & -0.01 & & & & & & \\
\hline J3CD & 612.93 & 0.84 & 0.71 & & & & & & \\
\hline J4CD & 616.84 & 1.69 & 1.42 & & & & & & \\
\hline J5AF & 619.84 & 2.22 & 1.86 & & & & & & \\
\hline J6AF & 623.75 & 3.86 & 3.25 & & & & & & \\
\hline J7CD & 626.74 & 5.26 & 4.42 & 0.01 & 0.00 & & & & \\
\hline J8CD & 630.66 & 4.97 & 4.17 & 0.01 & 0.00 & & & & \\
\hline J9BF & 633.27 & 4.08 & 2.74 & 0.81 & 0.81 & & & & \\
\hline J10BF & 635.89 & 4.25 & 1.95 & 1.92 & 1.93 & & & & \\
\hline D10 & 638.94 & 5.19 & 1.40 & 3.50 & 3.51 & & & & \\
\hline J11BD & 640.94 & 5.29 & 0.92 & 4.17 & 4.18 & -0.01 & -0.01 & & \\
\hline J12BD & 643.56 & 4.25 & 0.11 & 4.11 & 4.12 & -0.01 & -0.01 & & \\
\hline J13CF & 646.56 & 3.18 & 0.01 & 3.81 & 3.16 & 0.95 & 0.70 & & \\
\hline J14CF & 650.47 & 3.17 & 0.00 & 5.03 & 3.16 & 2.75 & 2.03 & & \\
\hline J15AD & 653.46 & 3.42 & 0.00 & 6.25 & 3.41 & 4.16 & 3.08 & & \\
\hline J16AD & 657.38 & 2.38 & 0.00 & 5.33 & 2.38 & 4.32 & 3.20 & & \\
\hline J17CF & 660.37 & 1.15 & -0.01 & 3.82 & 1.16 & 3.90 & 2.89 & 0.00 & 0.01 \\
\hline J18CF & 664.28 & 0.06 & -0.01 & 3.61 & 0.07 & 5.19 & 3.84 & 0.00 & 0.01 \\
\hline J19BD & 666.90 & -0.02 & -0.02 & 3.89 & 0.00 & 6.45 & 4.22 & 0.75 & 0.76 \\
\hline J20BD & 669.52 & -0.02 & -0.02 & 3.20 & 0.00 & 6.11 & 3.47 & 1.40 & 1.41 \\
\hline K1BFM & 674.57 & & & 0.95 & 0.00 & 3.92 & 1.03 & 2.49 & 2.50 \\
\hline $\mathrm{K} 2 \mathrm{BFM}$ & 677.19 & & & 0.09 & 0.00 & 4.00 & 0.10 & 3.80 & 3.80 \\
\hline $\mathrm{K} 3 \mathrm{CD}$ & 680.18 & & & 0.01 & -0.01 & 4.77 & 0.01 & 5.24 & 4.68 \\
\hline K4CD & 684.09 & & & 0.01 & 0.00 & 3.77 & 0.02 & 4.82 & 3.70 \\
\hline K5AF & 687.09 & & & & & 2.37 & 0.01 & 3.79 & 2.32 \\
\hline K6AF & 691.00 & & & & & 1.52 & 0.02 & 4.04 & 1.48 \\
\hline K7CD & 694.00 & & & & & 1.08 & 0.02 & 4.53 & 1.04 \\
\hline $\mathrm{K} 8 \mathrm{CD}$ & 697.91 & & & & & 0.09 & 0.02 & 3.36 & 0.07 \\
\hline K9BF & 700.53 & & & & & & & 2.16 & -0.01 \\
\hline K10BF & 703.14 & & & & & & & 1.53 & -0.01 \\
\hline D10 & 706.19 & & & & & & & 1.10 & -0.01 \\
\hline K11BD & 708.20 & & & & & & & 0.72 & -0.01 \\
\hline K12BD & 710.81 & & & & & & & 0.08 & -0.01 \\
\hline K13CF & 713.81 & & & & & & & 0.00 & 0.00 \\
\hline
\end{tabular}

\begin{tabular}{|c|c|}
\cline { 2 - 2 } \multicolumn{1}{c|}{} & J02L+ \\
\hline J02L+ & J08L- \\
\hline J08L & J12L \\
\hline$Y$ & $Y$ \\
\hline $\mathrm{mm}$ & $\mathrm{mm}$ \\
\hline 0.00 & -0.01 \\
\hline 0.01 & -0.01 \\
\hline 0.86 & 0.85 \\
\hline 1.71 & 1.70 \\
\hline 2.23 & 2.22 \\
\hline 3.88 & 3.87 \\
\hline 5.27 & 5.27 \\
\hline 4.98 & 4.97 \\
\hline 4.89 & 4.89 \\
\hline 6.17 & 6.17 \\
\hline 7.43 & 7.43 \\
\hline 9.45 & 9.46 \\
\hline 8.36 & 8.37 \\
\hline 6.98 & 6.03 \\
\hline 8.20 & 5.45 \\
\hline 9.67 & 5.50 \\
\hline 7.71 & 3.39 \\
\hline 4.97 & 1.07 \\
\hline 3.67 & -1.52 \\
\hline 3.87 & -2.59 \\
\hline 3.18 & -2.93 \\
\hline 0.93 & -2.99 \\
\hline 0.07 & -3.93 \\
\hline-0.02 & -4.79 \\
\hline-0.01 & -3.78 \\
\hline 0.00 & -2.37 \\
\hline 0.01 & -1.52 \\
\hline 0.01 & -1.07 \\
\hline 0.02 & -0.07 \\
\hline 0.02 & 0.01 \\
\hline 0.02 & 0.02 \\
\hline & \\
\hline
\end{tabular}


VERTICAL THREE BUMPS: J02L \& J02S

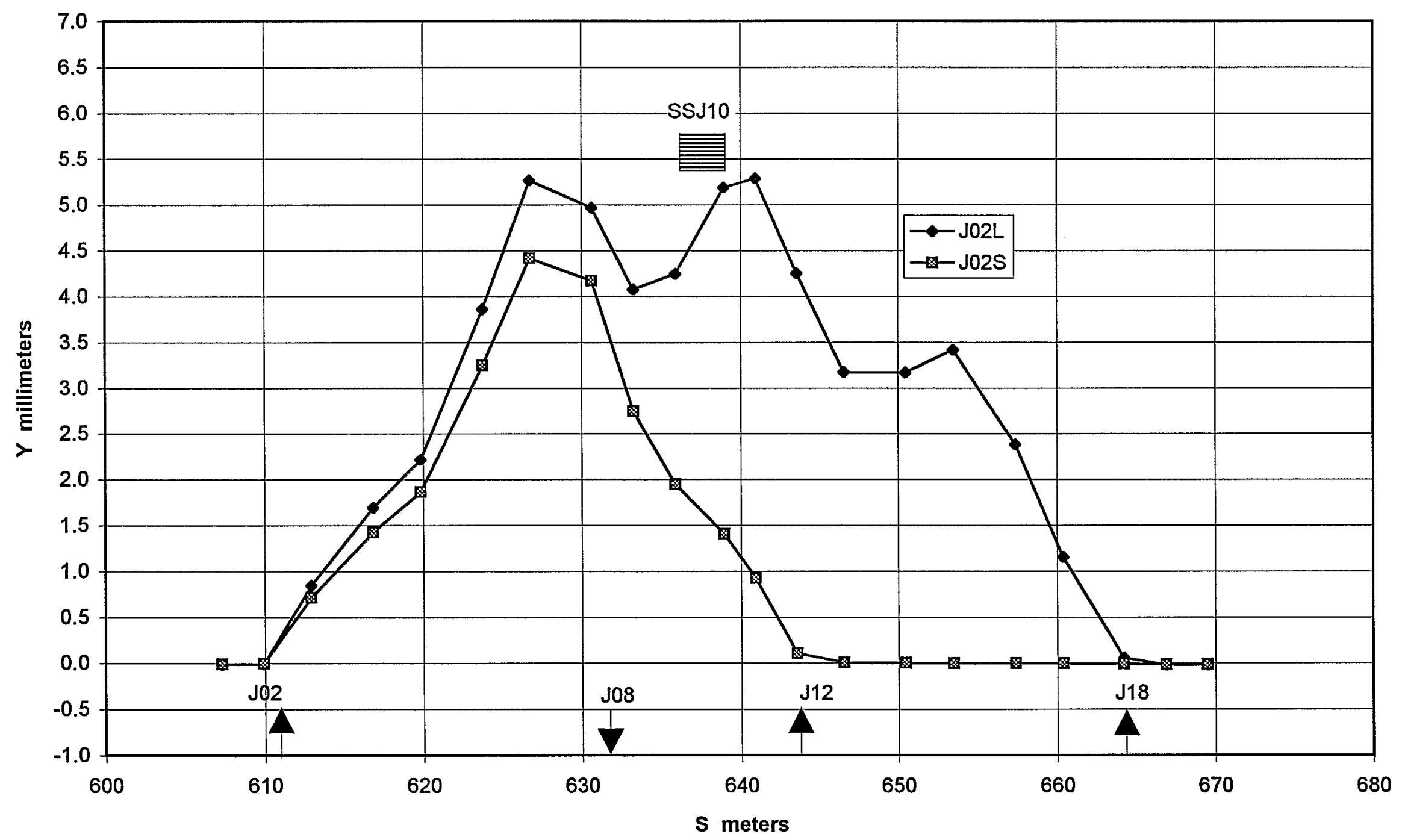

Figure 1 
VERTICAL THREE BUMPS: J08L \& J08S

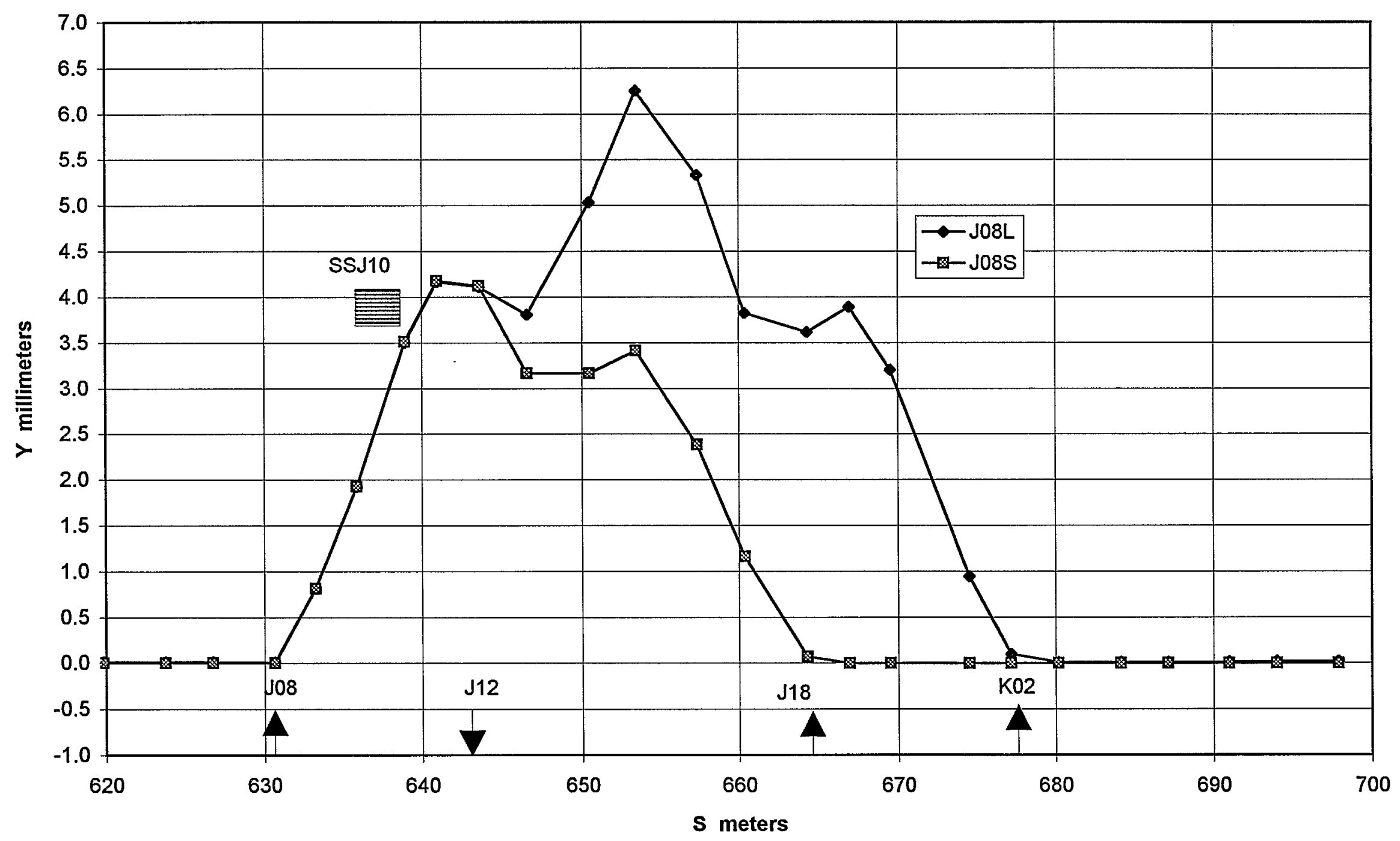

Figure 2 


\section{VERTICAL THREE BUMPS: J12L \& J12S}

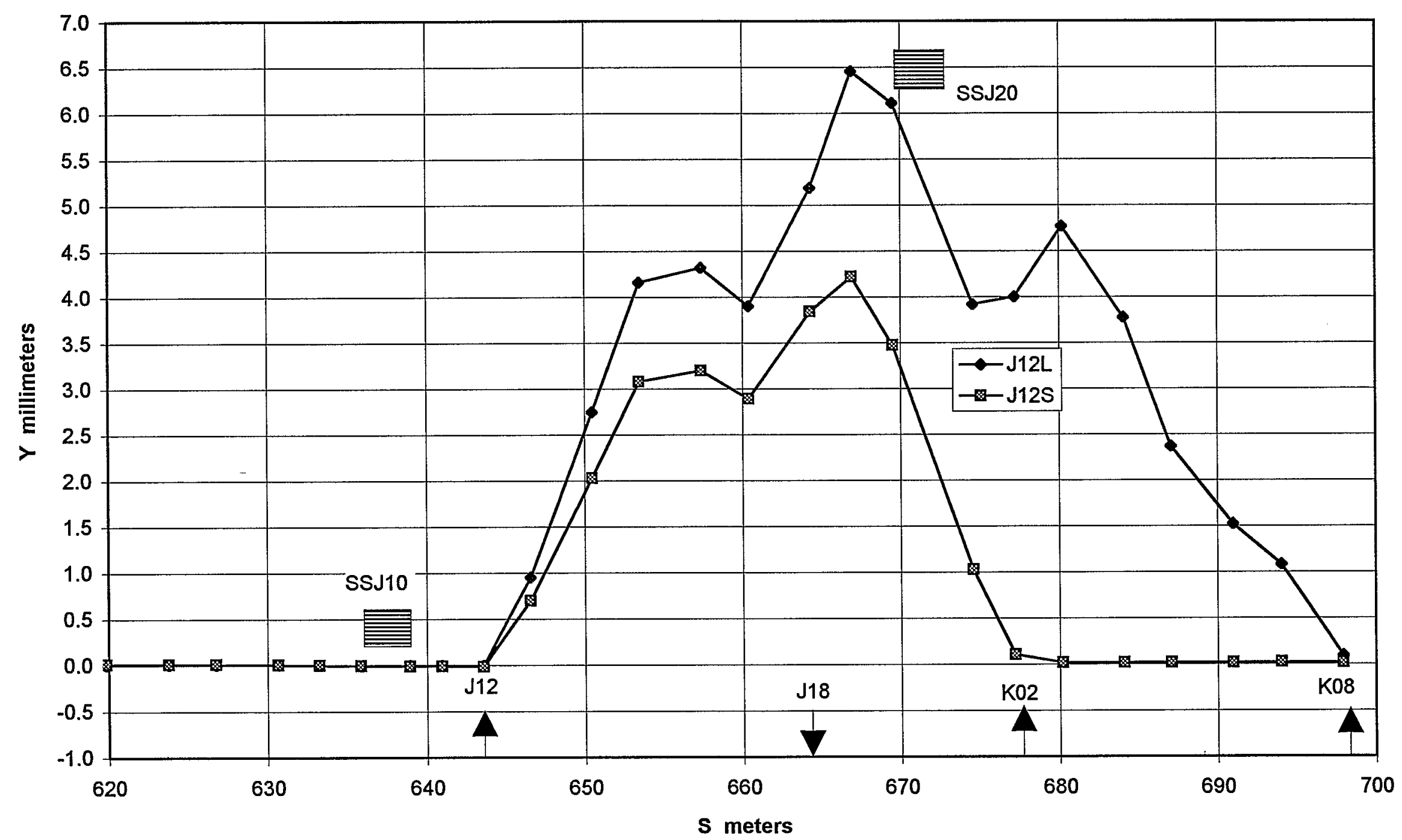

Figure 3 
VERTICAL THREE BUMPS: J18L \& J18S

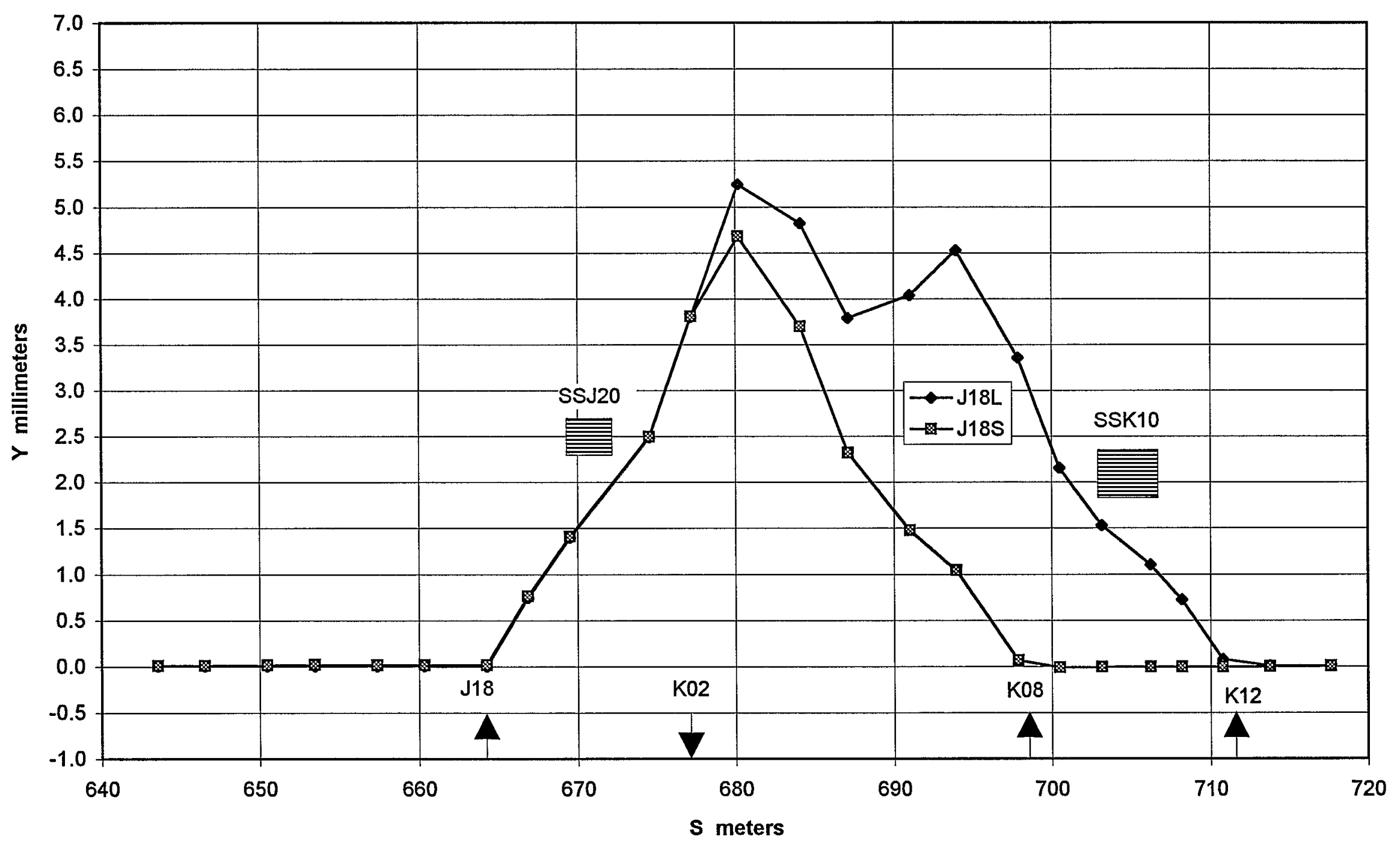


VERTICAL BUMP for J10: J02L + J08L - J12L

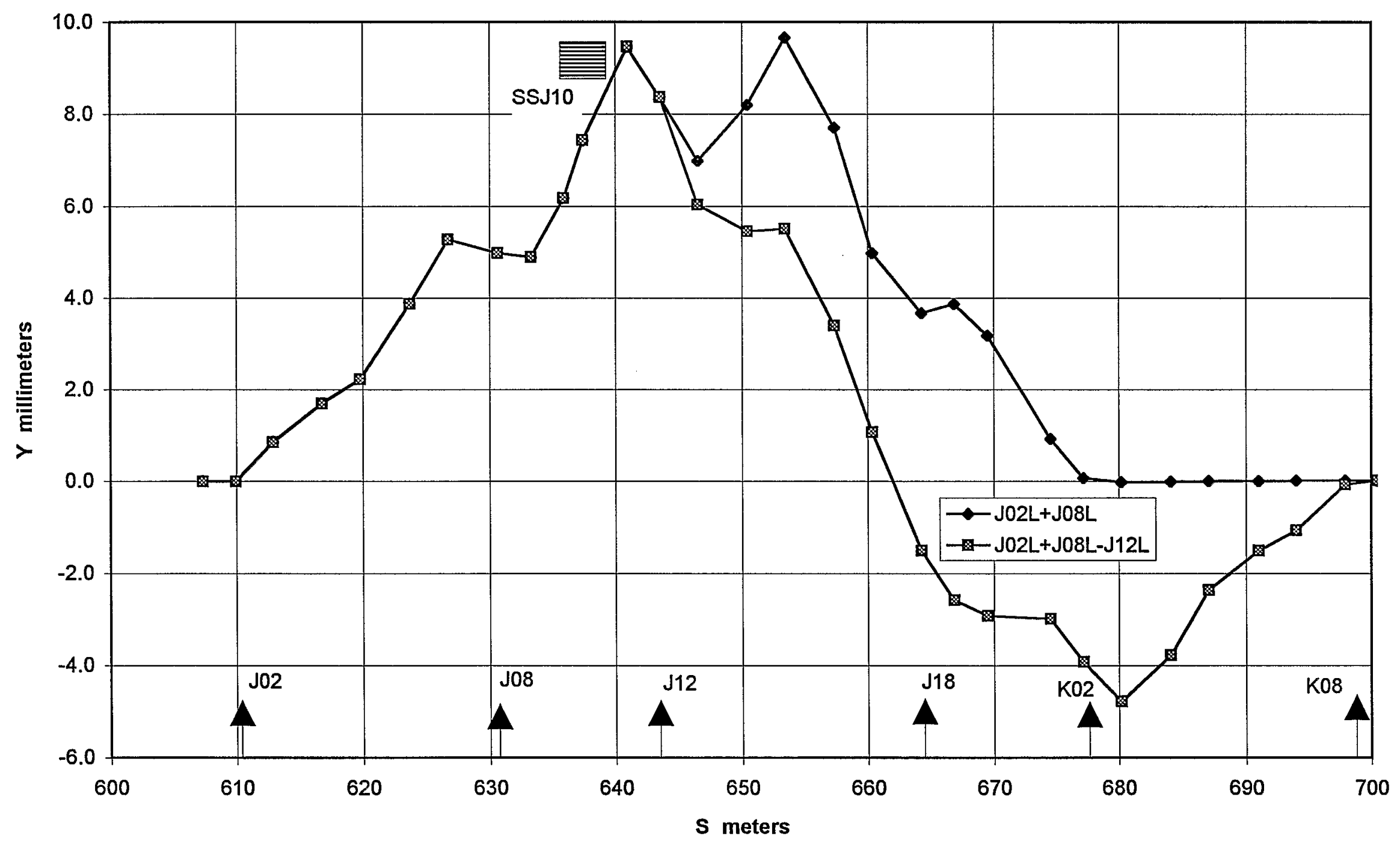

Figure 5 


\section{Appendix I}

\section{The Standard Injection AGS}

We define the standard injection AGS as having a momentum of $2.25 \mathrm{GeV} / \mathrm{c}$ and currents in both tune quad strings of 50 Amperes. This produces a lumpy AGS as tabulated in Table A1 and shown in Figures $A 1, A 2$, and A3. On the figures the arrows indicate the exit points of the main magnets. The results given are for Superperiod $J$, but the other superperiods are identical. The absolute value of the tune of the AGS has not been carefully cross calibrated against the MAD calculation and the present results may not agree exactly with reality, but they are in general very good. 
TABLE A1

THE STANDARD INJECTION AGS

MAD INPUT: $P=2.25 \mathrm{GeV} / \mathrm{c}, \mathrm{l}_{Q X}=50 \mathrm{AMPS}, \mathrm{l}_{Q Y}=50 \mathrm{AMPS}$

MAD OUTPUT: $Q_{X}=8.83903, Q_{Y}=8.892302$

\begin{tabular}{|c|c|c|c|c|c|c|c|c|c|c|}
\hline & & & \multicolumn{5}{|c|}{ HORIZONTAL } & \multicolumn{3}{|c|}{ VERTICAL } \\
\hline POS. & LEMENT & DIST & BETAX & ALFAX & MUX & $\overline{D X}$ & DPX & BETAY & ALFAY & MUY \\
\hline NO. & NAME & $\mathrm{m}$ & $\mathbf{m}$ & & [2PI] & $\mathrm{m}$ & & $\mathbf{m}$ & & [2PI] \\
\hline 630 & D10 & 605.307 & 18.463 & -1.483 & 6.629 & 1.971 & 0.111 & 10.983 & 0.947 & 6.669 \\
\hline 631 & IBF & 07.313 & 20.948 & 0.326 & 645 & 2.019 & -0.063 & 9.692 & -0.263 & 0.701 \\
\hline 632 & $2 S$ & 607.923 & 20.570 & 0.294 & 6.650 & 1.981 & -0.063 & 10.054 & -0.330 & .711 \\
\hline 633 & $2 \overline{B F}$ & 609.929 & 15.974 & 1.845 & 6.667 & 1.692 & -0.221 & 4.117 & -1.825 & 6.73 \\
\hline 634 & DPUE & 10.216 & 14.938 & 1.766 & 6.670 & 1.629 & -0.221 & 5.190 & -1.913 & $\overline{6.742}$ \\
\hline 635 & PUE J02 & 10.216 & 14.938 & 1.766 & 6.670 & 1.629 & -0.221 & 5.190 & -1.913 & 6.742 \\
\hline 0 & DVCJ02 & 610.216 & 14.938 & 1.766 & 6.670 & 1.629 & -0.221 & 5.190 & 1.913 & 6.742 \\
\hline 637 & D2TX & 610.539 & 13.828 & 1.677 & 6.673 & 1.558 & -0.221 & 16.456 & 2.012 & 6.745 \\
\hline 0 & $\overline{C D}$ & 12.927 & 10.180 & -0.011 & 6.707 & 1.261 & -0.033 & 21.805 & 0.017 & 6.765 \\
\hline 6 & $S Q$ & 613.298 & 10.202 & -0.047 & 6.713 & 1.249 & -0.033 & 21.824 & 0.034 & 6.767 \\
\hline 640 & QHFV & 688 & 10.301 & -0.206 & 6.719 & 1.239 & -0.019 & 21.757 & 0.204 & 6.770 \\
\hline 641 & QPOL & 614.079 & 10.477 & -0.245 & 6.725 & 1.232 & -0.019 & 21.605 & 0.185 & 6.773 \\
\hline 642 & $S Q$ & 450 & 10.673 & -0.283 & 6.731 & 1.225 & 19 & 21.475 & 0.167 & 6.776 \\
\hline 6 & $C D$ & 838 & & -2.226 & 6.761 & 1.386 & & 15.627 & 2.051 & 6.796 \\
\hline 644 & DUE & & & -2.331 & 6.764 & 1.431 & & 14.477 & & 6.799 \\
\hline 6 & PUE_J04 & & & -2.331 & 6.764 & 1.431 & & 14.477 & 1.955 & 107 \\
\hline & & & & -2.450 & 6.767 & 1.482 & & 13.251 & 1.848 & 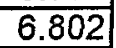 \\
\hline & & & & -0.113 & 6.783 & 1.671 & & 8.806 & & \\
\hline & & & 35 & -0.143 & 788 & 1.669 & & 8.596 & & \\
\hline & & & 35 & -0.173 & 2 & 1.668 & & 8.523 & & \\
\hline & & & & 2.350 & 8 & 1.469 & & 11.820 & 9 & \\
\hline & & & & .156 & 3 & 1.372 & & 63 & 78 & \\
\hline & & 44 & & .096 & 0 & 1.200 & & 28 & 53 & \\
\hline & & 78 & & .062 & 5 & 1.206 & & 34 & 77 & 342 \\
\hline$\underline{6}$ & & 33 & & .010 & 3 & 1.215 & & & 12 & 348 \\
\hline 6 & & 67 & & 0.024 & 59 & 1.220 & & 16 & -0.136 & 951 \\
\hline 56 & & 6 & & 2.024 & 36 & 1.461 & & 22 & 1.613 & .97 \\
\hline 7 & $\mathrm{UE}$ & 42 & & -2.109 & 888 & 1.516 & & 17 & 1.543 & .97 \\
\hline 8 & JE_ J08 & 42 & & -2.109 & 888 & 1.516 & & 13.817 & 1.543 & .977 \\
\hline 9 & 508 & 42 & & -2.109 & 888 & 1.516 & & 13.817 & 1.543 & 6.977 \\
\hline 30 & $\overline{T X}$ & 631.264 & 19. & -2.205 & 6.891 & 1.578 & 93 & 12.847 & 1.464 & 6.980 \\
\hline 661 & & 633.271 & 24.990 & -0.240 & 6.905 & 1.824 & 0.048 & 9.898 & 0.100 & 7.010 \\
\hline 662 & & 633.881 & 25.298 & -0.265 & 6.909 & 1.854 & 0.048 & 9.814 & 0.038 & 7.020 \\
\hline 663 & $\overline{B F}$ & 887 & 63 & 1.911 & 6.922 & 1.793 & 08 & 12.118 & -1.260 & 7.050 \\
\hline 664 & 0 & 637.411 & 16.435 & 1.585 & 6.935 & 1.628 & 0.108 & 16.452 & -1.585 & 7.067 \\
\hline 665 & 0 & 638.935 & 12.100 & 1.260 & 6.952 & 1.463 & -0.108 & 21.778 & -1.910 & 7.080 \\
\hline$\overline{6} \overline{66}$ & $\mathrm{BD}$ & 640.941 & 9.798 & -0.039 & 6.983 & 1.408 & 0.052 & 25.298 & 0.272 & 7.093 \\
\hline 667 & & 551 & 83 & -0.101 & 6.992 & 1.440 & 0.052 & 24.982 & 0.246 & 7.097 \\
\hline 668 & $\bar{D}$ & 58 & 2.838 & -1.466 & 7.022 & 1.715 & 0.226 & 19.703 & 2.211 & 7.111 \\
\hline 669 & UE & 845 & 00 & -1.537 & 7.025 & 1.780 & 0.226 & 18.458 & 2.125 & 7.114 \\
\hline 670 & UE_J12 & .845 & 13.700 & -1.537 & 7.025 & 1.780 & $\begin{array}{ll}0.226 \\
\end{array}$ & 18.458 & 2.125 & 7.114 \\
\hline 671 & DVCJ12 & 845 & 00 & -1.537 & 7.025 & 1.780 & 0.226 & 18.458 & 2.125 & 7.114 \\
\hline 672 & & 644.167 & 14.717 & -1.616 & 7.029 & 1.854 & 0.226 & 17.119 & 2.029 & 7.116 \\
\hline 673 & $3 \mathrm{CF}$ & 646.555 & 18.632 & 0.130 & 7.051 & 2.151 & 0.017 & 12.600 & 0.035 & 7.144 \\
\hline
\end{tabular}




\begin{tabular}{|c|c|c|c|c|c|c|c|c|c|c|}
\hline & & & \multicolumn{5}{|c|}{ HORIZONTAL } & \multicolumn{3}{|c|}{ VERTICAL } \\
\hline POS. & ELEMENT & DIST & ETAX & $\overline{A L F A X}$ & MUX & $\overline{D X}$ & DPX & BETAY & ALFAY & MUY \\
\hline NO. & NAME & $\bar{m}$ & $\bar{m}$ & & [2PI] & $\bar{m}$ & & $\overline{\mathbf{m}}$ & & [2PI] \\
\hline 674 & DSS & 646.989 & 18.529 & 0.107 & 7.055 & 2.158 & 0.017 & 12.585 & 0.000 & 7.149 \\
\hline 675 & SXH & 647.644 & 18.413 & 0.071 & 7.060 & 2.169 & 0.017 & 12.619 & -0.052 & 7.157 \\
\hline 676 & DSS & 648.079 & 18.362 & 0.047 & 7.064 & 2.176 & 0.017 & 12.679 & -0.086 & 7.163 \\
\hline 677 & J14CF & 650.466 & 13.814 & 1.678 & 7.087 & 1.951 & -0.200 & 17.516 & -2.123 & 7.190 \\
\hline 678 & DPUE & 650.753 & 12.874 & 1.599 & 7.090 & 1.894 & -0.200 & 18.761 & -2.213 & 7.192 \\
\hline 679 & UE J14 & 753 & 12.874 & 1.599 & 7.090 & 1.894 & -0.200 & 18.761 & -2.213 & 7.192 \\
\hline 680 & D2LX & 076 & 11.872 & 1.509 & 7.094 & 1.829 & -0.200 & 20.221 & -2.315 & 7.195 \\
\hline 68 & & & & -0.009 & 7.134 & 1.622 & 0.023 & 25.849 & 0.180 & 7.211 \\
\hline & & & 670 & -0.098 & 7.148 & & 0.023 & & 0.150 & 7.216 \\
\hline & & & & 187 & 7.1 & 1.6 & .023 & & 0.119 & 7.220 \\
\hline & & & 32 & 866 & 7.199 & & 257 & & 2.418 & 7.237 \\
\hline 6 & & & & -2.071 & 7.206 & 2. & .257 & 390 & 2.195 & 7.243 \\
\hline 36 & & & & -0.169 & 7.225 & 2.4 & .011 & 770 & .280 & 7.274 \\
\hline & & 43 & 21. & -0.186 & 7.228 & 2.4 & 11 & 76 & .242 & 7.279 \\
\hline & & & 21.868 & 0.052 & 7.231 & 2.469 & -0.018 & & 0.083 & 7.285 \\
\hline 6 & & 25 & & .034 & 7.234 & 2.462 & -0.018 & & 0.044 & 7.292 \\
\hline 69 & & 66 & & .017 & 7.236 & 2.455 & -0.018 & & 0.007 & 7.297 \\
\hline & & 66 & & 2.011 & 7.256 & 2.1 & -0.261 & 13.717 & -1.668 & 7.331 \\
\hline 69 & & & & 1.923 & 7.258 & 2.039 & -0.261 & 14.697 & -1.747 & 7.335 \\
\hline 693 & UE_J18 & 664 & & 1.923 & 7.258 & 2.039 & -0.261 & 14.697 & -1.747 & 7.335 \\
\hline 694 & DVCJ18 & & & 1.923 & 7.258 & 2.039 & -0.261 & 14.697 & -1.747 & 7.335 \\
\hline 695 & D2TX & 66 & 32 & 1.825 & 7.262 & 1.955 & -0.261 & 15.853 & -1.836 & 7.338 \\
\hline 696 & J19BD & 66 & 10.076 & 0.327 & 7.290 & 1.631 & -0.067 & 20.436 & -0.296 & 7.355 \\
\hline 697 & & 667.509 & 9.719 & 0.260 & 7.300 & 1.590 & -0.067 & 20.816 & -0.329 & 7.360 \\
\hline 698 & J20BD & 669.515 & 11.031 & -0.955 & 7.332 & 1.634 & 0.111 & 18.357 & 1.473 & 7.376 \\
\hline 699 & $\overline{D 1}$ & 671.039 & 14.344 & -1.219 & 7.351 & 1.802 & 0.111 & 14.269 & 1.210 & 7.391 \\
\hline 700 & D10 & 672.563 & 18.463 & -1.483 & 7.366 & 1.971 & 0.111 & 10.983 & 0.947 & 7.410 \\
\hline
\end{tabular}


THE STANDARD INJECTION AGS: BETAY

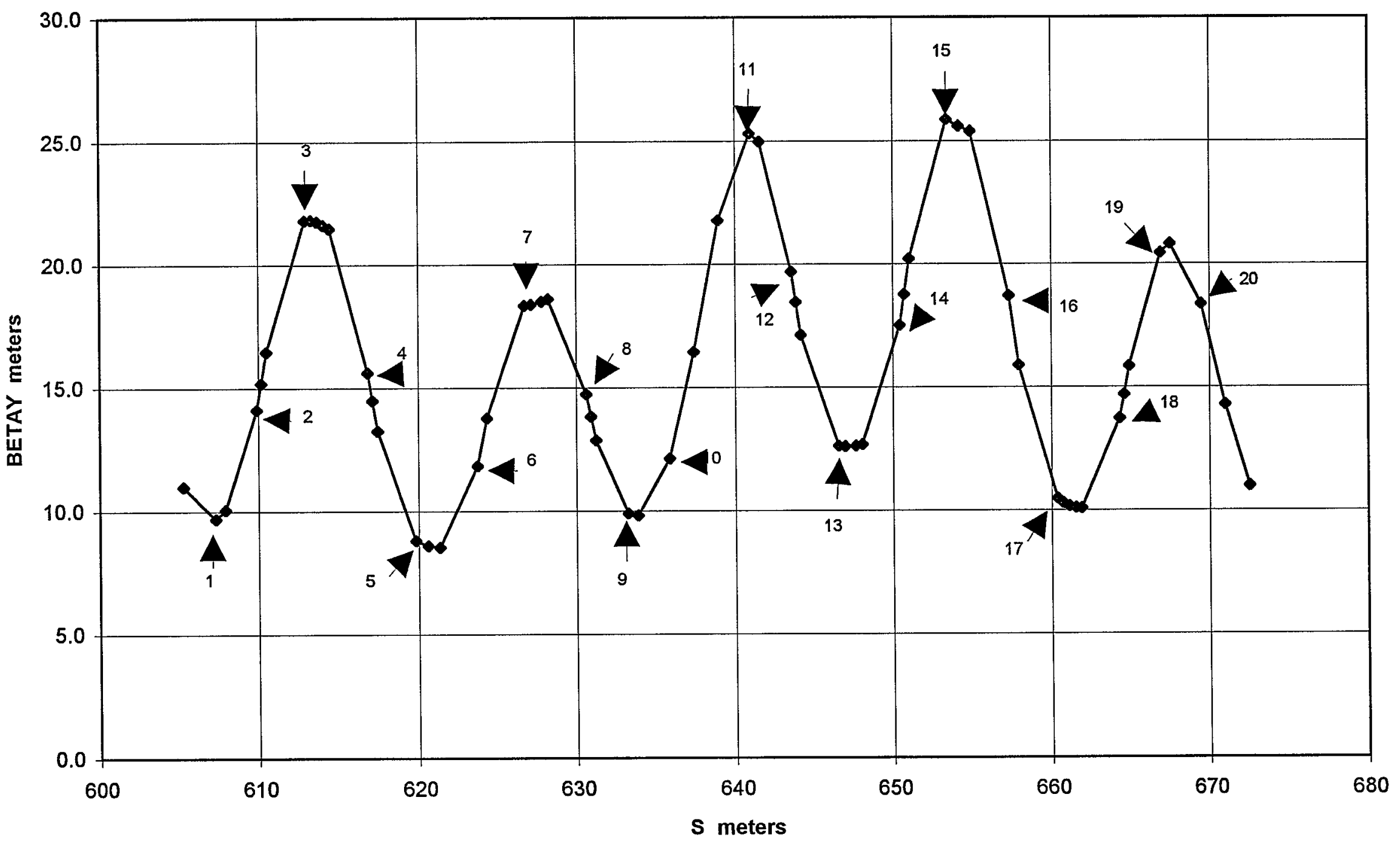


THE STANDARD INJECTION AGS: BETAX

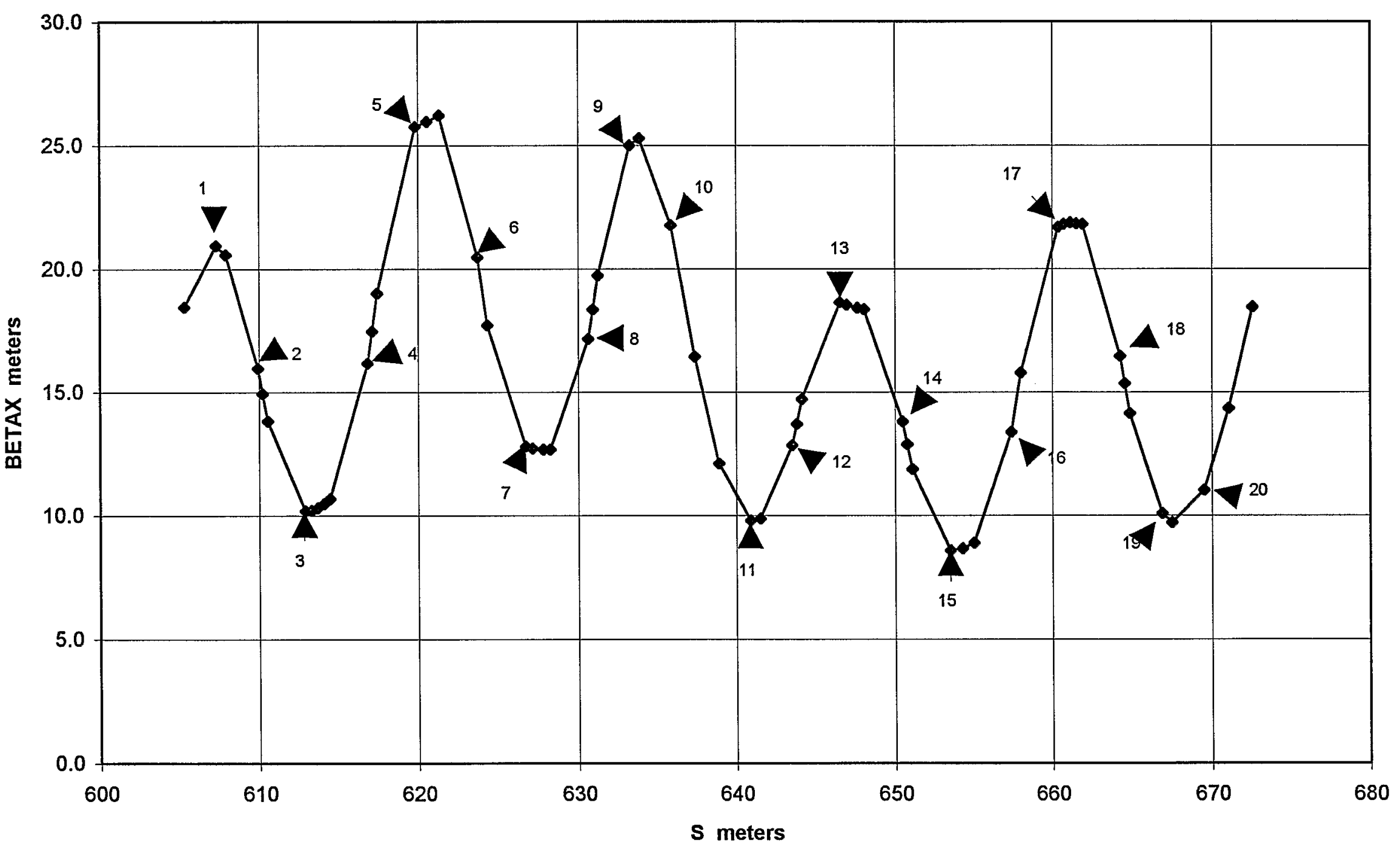


THE STANDARD INJECTION AGS: HORIZONTAL DISPERSION

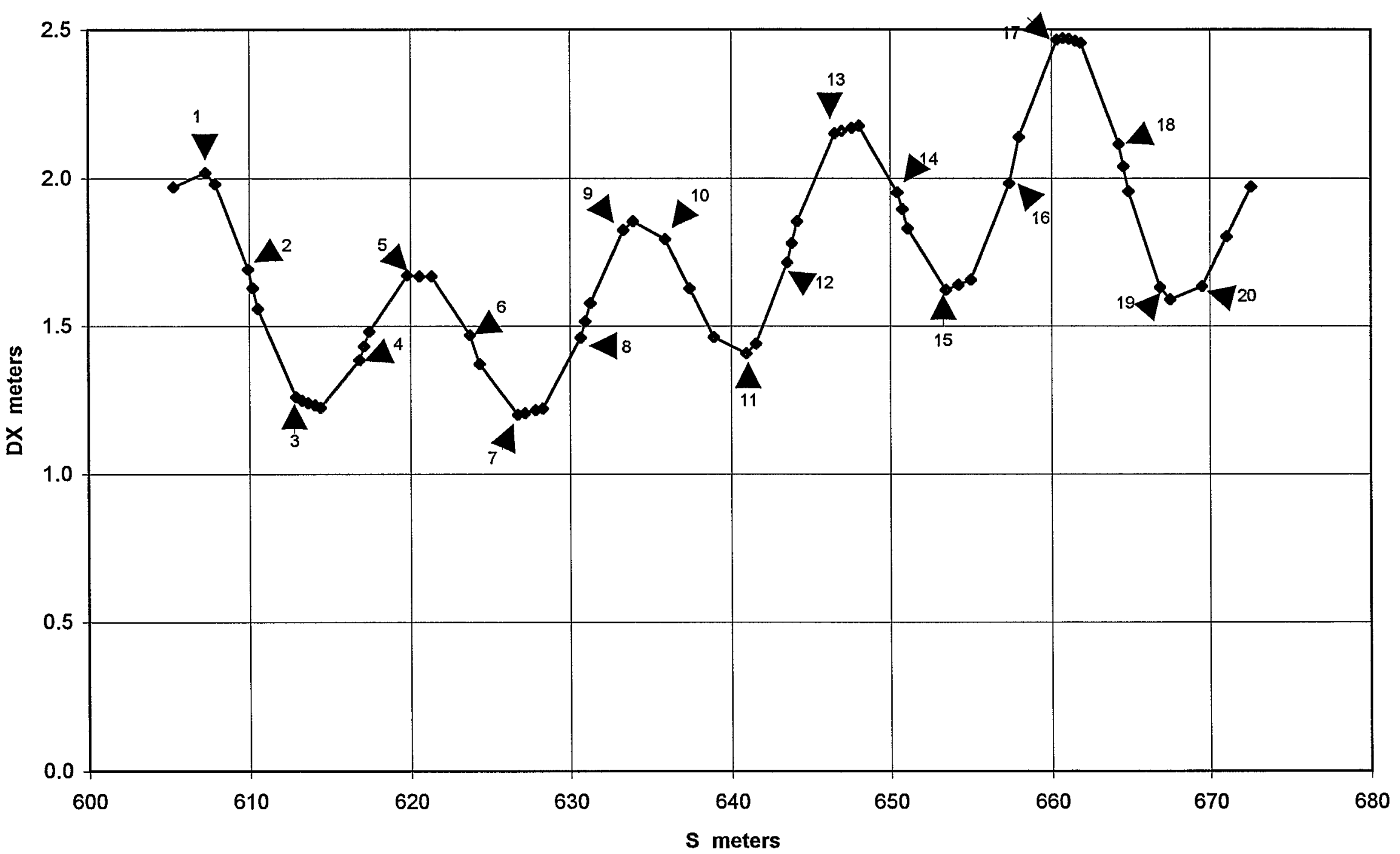

